\title{
OPEN Authenticity control of pine sylvestris essential oil by chiral gas chromatographic analysis of $\alpha$-pinene
}

\author{
Martina Allenspach ${ }^{1}$, Claudia Valder ${ }^{2}$, Daniela Flamm² \& Christian Steuer ${ }^{1 凶}$ \\ Numerous terpenes present in essential oils (EOs) display one or more chiral centers. Within the \\ same genus the enantiomeric ratio of these compounds can be different. Thus, the determination of \\ enantiomers is a valuable tool to evaluate authenticity and quality of EOs. In here, the terpene profile \\ of primary and commercial pine EOs was analyzed by conventional and chiral gas chromatography \\ coupled to a flame ionization detector. The enantiomeric excess of $( \pm)$ - $\alpha$-pinene was determined and \\ significant differences between primary and commercially available EOs were observed. Primary \\ EOs of Pinus sylvestris L. showed a positive enantiomeric excess of (+)- $\alpha$-pinene whereas commercial \\ EOs labeled as $P$. sylvestris $L$. exhibited an enantiomeric excess of $(-)$ - $\alpha$-pinene. Thus, chiral \\ analysis provides useful information on the authenticity of pine EOs and allows to uncover possible \\ mislabeling, the use of the wrong herbal substance and sources of adulteration in pine oil.
}

Essential oils (EOs) defined according to the European Pharmacopeia (Ph. Eur.) are odorous natural products obtained by steam distillation, dry distillation or a suitable mechanical process. Ph. Eur. allows further modifications of an EO, such as rectification ${ }^{1}$. Currently, 34 EOs from different medical plants are described and monoand sesquiterpenes are the main metabolites found in these herbal preparations. Mono- and sesquiterpenes may show chiral characters. However, a suitable test on chirality is not yet defined for pine EO in the Ph. Eur ${ }^{1,2}$. But determination of chiral characters in a pharmacological active product might be crucial, since enantiomers could differ in their interaction with their biological target ${ }^{3}$. Pine EOs show antibacterial activity in vitro and are used as therapeutic agents in numerous products ${ }^{4}$. As reported previously, $(+)$ - $\alpha$-pinene and $(+)-\beta$-pinene-main constituents of pine Eos-exhibit antimicrobial and anti-inflammatory activity ${ }^{5,6}$. In contrast, negative congeners show insecticidal toxicity ${ }^{5,7}$.

Within the same genus the enantiomeric ratio of main analytes differs from species to species ${ }^{2,8-10}$. In recent years, numerous research groups reported possible chiral impurities in EOs e.g., lavender, bergamot, balm, rose or peppermint $\mathrm{EO}^{2,10,11}$. Thus, chiral gas chromatography (GC) analysis is applied to detect the use of the wrong herbal substance or intentional adulteration ${ }^{12,13}$.

According to $\mathrm{Ph}$. Eur, an industrial EO can be deterpenated (removal of monoterpene hydrocarbons), desesquiterpenated (removal of sesquiterpene hydrocarbons), rectified or " $\mathrm{x}$ "-free EOs (removal of compound $\mathrm{x})^{1}$. Sesquiterpenes are removed due to their bitter taste and to their potential reduced solubility. During rectification primary EOs are usually re-distilled without water under vacuum to eliminate unwanted compounds with an undesirable odor or with allergic or toxic potential. However, conversion of enantiomeric centers or elimination of enantiomers is not observed ${ }^{12}$.

In order to guarantee appropriate quality of therapeutically used herbal substances the establishment of reliable and traceable qualities becomes more and more important. The supply chain for EOs usually involves many steps during collection of the starting material to the finished distilled product. Possible supply chain issues affect the quality of the final product. Especially, collection activities in non-defined agricultural environment often result in quality issues arising from confusion of closely related medicinal plants ${ }^{12,14}$. Chiral analysis therefore offers a unique tool to verify potential treatment other than that defined in the Ph. Eur. during supply chain assessment.

${ }^{1}$ Institute of Pharmaceutical Sciences, ETH Zürich, 8092 Zurich, Switzerland. ${ }^{2}$ Systema Natura GmbH, Konrad-Zuse-Ring 8, 24220 Flintbek, Germany. ${ }^{\varpi}$ email: christian.stever@pharma.ethz.ch 

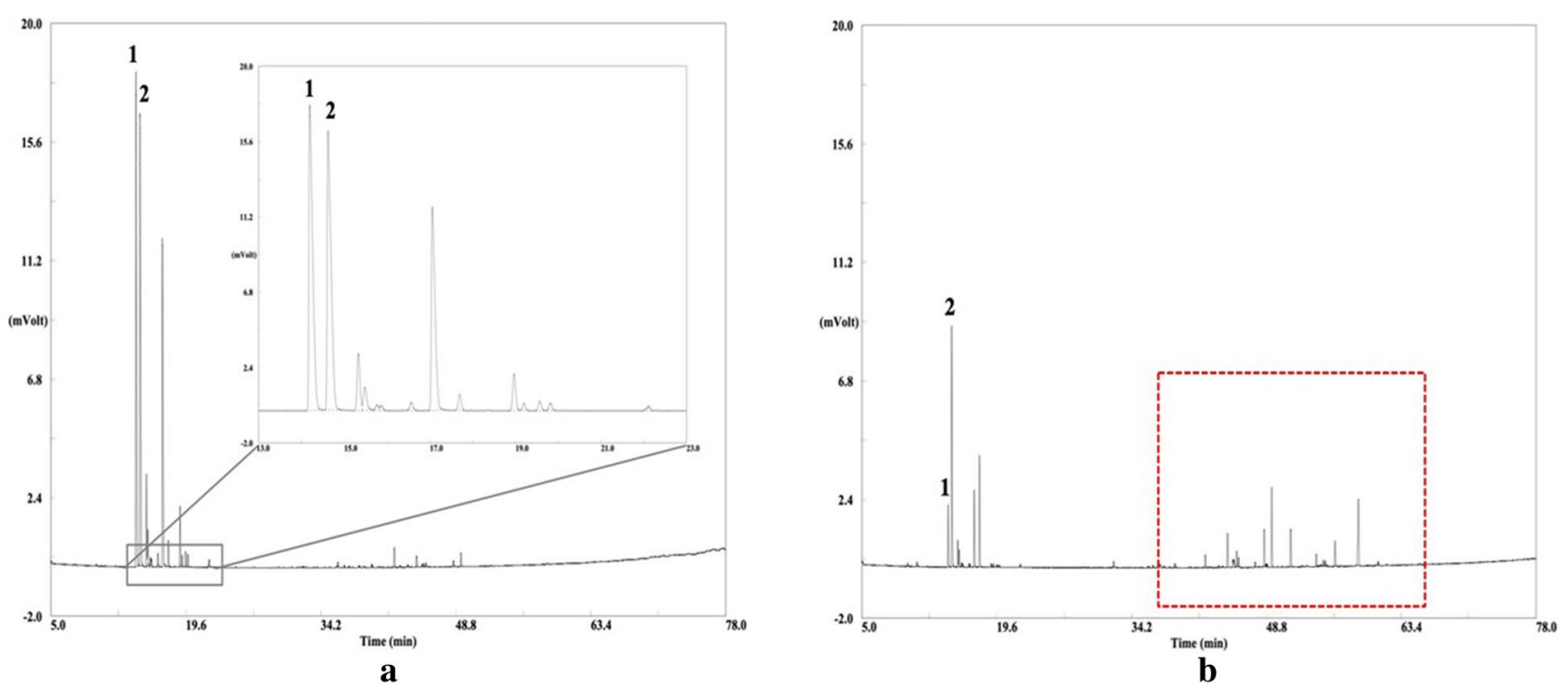

Figure 1. (a) Chromatographic profile obtained by chiral gas chromatography-flame ionization detector of a commercial essential oil of $P$. sylvestris (1) and (b). primary essential oil of $P$. sylvestris (36) with (-)- $\alpha$-pinene (1, RT: 14.3 min, RI cal: 977, RI lit: 977) and (+)- $\alpha$-pinene (2, RT: $14.7 \mathrm{~min}$, RI cal: 983, RI lit: 980). The red square indicates the distinct sesquiterpene profile, which was lacking in commercial EOs.

The total evaluation of a correct supply chain including unchangeable analytical markers as well as traceable information on the exact origin, harvest and storage of materials as defined in the good agricultural and collection practices $(\mathrm{GACP})^{12}$ are therefore a perfect combination in the frame of a successful herbal material qualification.

In case of EOs of Pinus sylvestris L. (P. sylvestris) three registered suppliers (European Chemicals Agency [ECHA]) import the EOs from different locations worldwide to provide them to local purchasers ${ }^{15}$.

Previously, our group investigated the chromatographic profiles of primary EOs of P. sylvestris obtained from traceable authentic plant material and developed a partial least squares discriminant analysis (PLS-DA) model for the correct taxonomic classification of closely related pine species ${ }^{4}$. The present work evaluates the chiral gas chromatographic-flame ionization detector (GC-FID) profile of primary and commercially available pine EOs as additional tool for authenticity control. Our results clearly demonstrate that for comprehensive quality control the enantiomeric ratio of at least the major terpenes in pine EOs is of high importance.

\section{Results and discussion}

Commercial EOs of $P$. sylvestris were obtained from the three registered suppliers for P. sylvestris EO (1-4, European Chemicals Agency [ECHA], Supplementary Information, Table A1) ${ }^{15}$ and local providers, respectively. In general, the chromatographic profile of a primary pine EOs from assigned and traceable pine trees (Supplementary Information, Table A2) consists of monoterpenes, sesquiterpenes and their oxygenated derivatives ${ }^{4}$. All commercial essential oils fulfilled the criteria given by the $\mathrm{Ph}$. Eur. for pine sylvestris oil. Our results clearly indicated, that commercial EOs were predominantly composed of monoterpene hydrocarbons with a-pinene as dominant analyte. However, commercial EOs of $P$. sylvestris showed significant different sesquiterpene pattern (Fig. 1a, Supplementary Information, Table A3) compared to primary EOs of the corresponding species (Fig. 1b, Supplementary Information, Table A4). A possible reason for the reduced sesquiterpene profile could have been modification of primary EOs e.g. by rectification ${ }^{1}$. One may speculate, if the low amount of minor compounds is the result of further dilution. Dilution with turpentine oil or turpentine oil based substances is a well-known adulteration in pine $\mathrm{EOs}^{12,16}$.

As illustrated in Table 1, the previously developed PLS-DA model was applied for taxonomic identification of commercial EOs of $P$. sylvestris ${ }^{4}$. Although they were labeled as $P$. sylvestris, none of them were classified as $P$. sylvestris. The commercial EOs remained either unclassified $(n=6)$ or were classified as EO derived from $P$. nigra $(n=5)$. Subsequently, an additional analytical tool is required to detect possible adulterations. Thus, all commercial and primary pine essential oils were analyzed by chiral GC-FID. Enantiomers of $\alpha$-pinene (Fig. 2) were baseline separated with a resolution of $R_{s}=2.1$ (Fig. 1a). In commercial and primary pine EOs, $\alpha$-pinene was present in both enantiomeric forms (+/-).

The dominant enantiomer in P. sylvestris of different origin was (+)-a-pinene (Fig. 3a). Until recently, only one research group reported an excess of $(+)$ - $\alpha$-pinene in EOs derived from $P$. sylvestris ${ }^{17}$. P. cembra showed the same dominant enantiomer as $P$. sylvestris (significantly different with $p<0.0001$ : $^{* * *}$ ) whereas the enantiomer (-)- $\alpha$-pinene was predominant in $P$. nigra and $P$. mugo (significantly different with $p<0.01$ : ${ }^{\star *}$ ), commercial EOs and turpentine oils. Only in three P. silvestris samples, a minor excess of (-)-a-pinene was detected. Our preliminary results showed that primary pine EOs were classified into their taxonomic specification by PLS-DA, a supervised chemometric method ${ }^{4}$. However, adding chiral information of $\alpha$-pinene to the existing 39 variables and fourth root calculation as data preprocessing, unsupervised principle component analysis (PCA) was sufficient to uncover data patterns in GC-FID chromatograms. Three-dimensional (3D) PCA allowed a clear 


\begin{tabular}{|c|c|c|c|}
\hline Commercial EOs of $P$. sylvestris & Predicted class & Enantiomer & Enantiomeric excess (\%) \\
\hline 1 & P. nigra & (-)- $\alpha$-pinene & $3.1 \pm 0.0$ \\
\hline 2 & Not classified & (-)-a-pinene & $19.8 \pm 0.0$ \\
\hline 3 & Not classified & (-)-a-pinene & $78.4 \pm 0.1$ \\
\hline 4 & P. nigra & (-)-a-pinene & $76.0 \pm 0.0$ \\
\hline 5 & Not classified & (-)-a-pinene & $74.3 \pm 0.0$ \\
\hline 6 & Not classified & $(-)$ - $\alpha$-pinene & $82.4 \pm 0.0$ \\
\hline 7 & Not classified & (-)-a-pinene & $69.1 \pm 0.0$ \\
\hline 8 & P. nigra & (-)-a-pinene & $76.1 \pm 0.1$ \\
\hline 9 & P. nigra & (-)-a-pinene & $66.7 \pm 0.1$ \\
\hline 10 & P. nigra & (-)-a-pinene & $8.6 \pm 0.1$ \\
\hline 11 & Not classified & $(-)$ - $\alpha$-pinene & $83.0 \pm 0.0$ \\
\hline
\end{tabular}

Table 1. Taxonomic classification of commercial essential oils of $P$. sylvestris by the developed PLS-DA model ${ }^{4}$ and the enantiomeric excess of $(-)-\alpha$-pinene. Data were expressed as mean \pm standard deviation $(n=3)$.<smiles>CC1=CC[C@@H]2C[C@H]1C2(C)C</smiles>

1

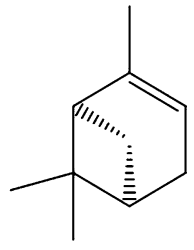

2

Figure 2. Chemical structures of the two enantiomers: (-)- $\alpha$-pinene (1) and (+)- $\alpha$-pinene (2).

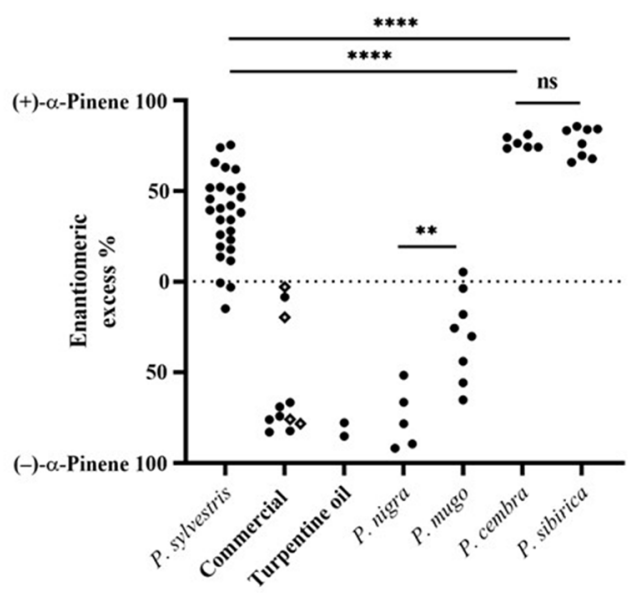

Essential oils

$\mathbf{a}$

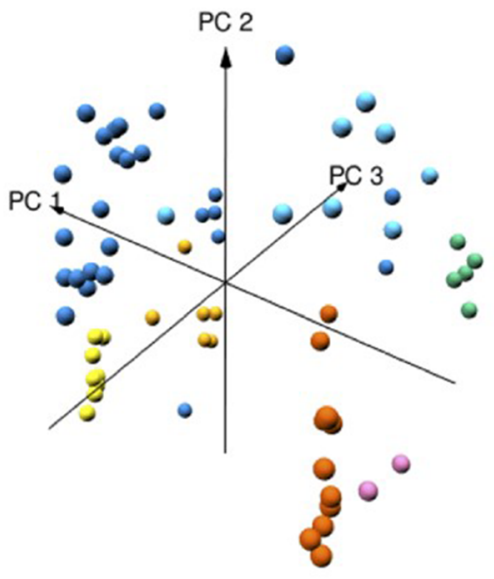

b

Figure 3. (a) The enantiomeric excess values (\%) of ( \pm )- $\alpha$-pinene of primary, commercial pine EOs (ECHA products in diamond shape) and turpentine oil. Significance of the enantiomeric excess values (\%) of $(+)$ - $\alpha$-pinene and (-)- $\alpha$-pinene were tested using Welch's ANOVA test followed by Games-Howell's multiple comparisons post-hoc test with $p<0.01\left(^{* *}\right), p<0.0001\left(^{* * *}\right)$ and ns: not significant. (b) The 3D score plot of the first three principal components PC1 (45.5\%), PC2 (15.2\%) and PC3 (9.5\%) for primary pine and commercial EOs based on their chemical composition obtained by the conventional GC-FID and ( \pm )- $\alpha$-pinene. EOs are colored based on their taxonomic specification and commercial origin, respectively.

taxonomic separation between primary pine and commercial EOs (Fig. 3b) but also in-between primary oils of different pines based on the first three principal components (PC1, PC2 and PC3) explaining 70.2\% of the total variance. However, PCA revealed chemical similarity of commercial EOs and turpentine oils emphasizing possible dilution with turpentine oil based substances. The corresponding biplot is presented in the Supplementary Information, Figure A1. 
The majority of the commercial EOs showed the opposite enantiomeric ratio of $( \pm)$ - $\alpha$-pinene compared to primary EOs of $P$. sylvestris and exhibited a high ee of (-)- $\alpha$-pinene (Fig. 3a). This is in-line with a previous report, which exhibited the enantiomeric content in commercially manufactured pine EOs ${ }^{18}$. Our data showed, that the commercial EOs were distinguished into two groups. Most of the commercial EOs belonged to group 1 (65.0-85.0\%) whereas the ee of the second group ranged from $0.0-20.0 \%$. Although the second group showed similar ee values as observed for primary EO's the main part of all commercial oils is significant different regarding the ee. According to our data, a threshold of e.g. $17.5 \%$ ee for (-)- $\alpha$-pinene would allow identification of a commercial essential oil (specificity: 1.0; sensitivity: 0.82 ). Additionally, commercial EOs could be assigned to one of the three registrants in terms of their ee of (-)- $\alpha$-pinene (Fig. 3a). Finally, the therapeutic benefit of these EOs is questionable, since (+)- $\alpha$-pinene is responsible for the antimicrobial and anti-inflammatory effects, but they predominantly consisted of (-)- $\alpha$-pinene ${ }^{5,6}$.

A possible reason for the high ee of $(-)$ - $\alpha$-pinene could have been an intended or unintended confusion in the raw material. Since $P$. nigra shares the same habitat and shows similar morphological properties to $P$. sylvestris. Lack of botanical knowledge could have led to misidentification and confusion in the raw material. Obviously, commercial EOs showed a similar enantiomeric pattern compared to turpentine oil. In turpentine oil-byproduct of paper industry-, also an excess of (-)- $\alpha$-pinene was found (Fig. 3a) $)^{19}$. Based on our results, we are convinced that chiral analysis is helpful to uncover possible mislabeling and sources of adulteration in pine essential oils. Additionally, enantiomeric distribution of $( \pm)$ - $\alpha$-pinene can be used to assign the commercial EOs of second suppliers to one of the three registered ECHA-distributors. Another example reinforced chiral analysis as an additional tool to determine authenticity of primary EOs. Primary EOs (60-67), labeled as $P$. sylvestris, remained unclassified and exhibited a high ee of (+)-a-pinene (Fig. 3a). Considering their Siberian origin, they were assigned as $P$. sibirica, known as a subspecies of $P$. cembra. EOs of $P$. sibirica and $P$. cembra were not significantly different in terms of their ee of $(+)$ - $\alpha$-pinene $(p>0.05$, ns). However, difference to $P$. sylvestris was highly significant $\left(p<0.0001:^{* * * *}\right)$.

To ensure the correct identification of pine species used as starting material international standards are recommended. Good Agricultural and Collection Practice for medicinal and aromatic plants (GACP) provides unique guidelines on correct collection of raw material. GACP controls the quality during cultivation, harvesting, processing, labeling and storage to obtain herbal products of correctly identified authentic plants for therapeutic and recreational use $\mathrm{e}^{12,20}$.

In conclusion, for the evaluation of the correct herbal substance and preparation in the field of pine EOsnext to a traceable supply chain - the unique chromatographic quality including analytical markers is crucial. Chromatographic profile obtained by GC-FID is able to distinguish between primary and commercial pine EOs. Lacking sesquiterpene patterns in further treated commercial oils might be explained by rectification. Chiral analysis however provides additional significant information on the authenticity of pine EOs and allows to uncover possible mislabeling, the use of wrong herbal substances and/or adulteration within the supply chain.

\section{Materials and methods}

Material. Commercially available EOs of $P$. sylvestris $(n=11,1-11)$ and turpentine oils $(n=2,12-13)$ were purchased from different suppliers. A detailed overview and used in-house codes are given in the Supplementary Information, Table A1. Primary Pine EOs were obtained from needles and twigs collected from $P$. sylvestris $(n=27,14-40)$, Pinus cembra L. (P. cembra) $(n=6,41-46)$, Pinus mugo TURRA (P. mugo) $(n=8,47-54)$, Pinus nigra J. F. ARNOLD (P. nigra) $(n=5,55-59)$ and Pinus sibirica DU TOUR $(P$. sibirica $)(n=8,60-67)$. Plant material was classified by macroscopic botanical identification. A detailed overview of the used in-house codes, GPS coordinates and harvesting times can be found in the Supplementary Information, Table A2. The EO of freshly cut (pieces of $1 \mathrm{~cm}$ ) needles and twigs was obtained by industrial distillation.

GC-FID analysis. The GC-FID analysis was performed using a Thermo Fisher Scientific Focus gas chromatograph (Thermo Fisher Scientific, Waltham, Massachusetts, USA) equipped with a DB-wax capillary column $(30 \mathrm{~m} \times 0.25 \mathrm{~mm}$ i.d., film thickness $0.25 \mu \mathrm{m})$. The temperature of the injection was $220^{\circ} \mathrm{C}$. The injection volume was $1 \mu \mathrm{l}$ (Autosampler AI3000, Thermo Fisher Scientific) using a split ratio of 1:50 with a split flow of $75 \mathrm{ml} / \mathrm{min}$. Helium was used as carrier gas at a constant flow rate of $1.5 \mathrm{ml} / \mathrm{min}$. The oven temperature was kept at $65^{\circ} \mathrm{C}$ for $10 \mathrm{~min}$ and then heated to $220^{\circ} \mathrm{C}$ with $5^{\circ} \mathrm{C} / \mathrm{min}$ and kept constant at $220^{\circ} \mathrm{C}$ for $9 \mathrm{~min}$. The temperature of the detector was $250^{\circ} \mathrm{C}$. Primary EOs diluted in heptane and commercial EOs (ten-fold diluted with heptane) were analyzed using the relative percentages of the individual components based on the FID response (peak area). The data were acquired with Chrom Card Trace Focus GC (Thermo Fisher scientific, version 2.9).

The chiral GC-FID analysis (Thermo Fisher Scientific, Waltham, Massachusetts, USA) was performed using a Thermo Fisher Scientific Trace 1300 gas chromatograph equipped with a BGB 176 SE capillary column $(30 \mathrm{~m} \times 0.25 \mathrm{~mm}$ i.d., film thickness $0.25 \mu \mathrm{m})$. The chiral column consists of $30 \% 2$,3-dimethyl-6-tertbutyldimethylsilyl- $\beta$-cyclodextrin dissolved in SE-52 (5\% phenyl-, $95 \%$ methylpolysiloxane). The temperature of the injection was $220^{\circ} \mathrm{C}$. The injection volume was $1 \mu \mathrm{l}$ (commercial EOs) or $2 \mu \mathrm{l}$ (primary EOs) (Autosampler AI3000, Thermo Fisher Scientific) using a split ratio of 1:70 with a split flow of $28 \mathrm{ml} / \mathrm{min}$. Helium was used as carrier gas at a constant flow rate of $2.5 \mathrm{ml} / \mathrm{min}$. The oven temperature was kept at $50{ }^{\circ} \mathrm{C}$ for $3 \mathrm{~min}$ and then heated to $200^{\circ} \mathrm{C}$ with $2{ }^{\circ} \mathrm{C} / \mathrm{min}$. The temperature of the detector was $250^{\circ} \mathrm{C}$. Peaks were identified by comparing retention times (RT) with reference substances. (-)- $\alpha$-Pinene was purchased from Fluka Chemie GmbH (Buchs, Switzerland). (+)- $\alpha$-Pinene was obtained from Sigma-Aldrich (St. Louis, MO, USA). Retention indices (RI cal) were calculated according to the van den Dool and Kratz equation with the RI in the literature (RI lit) ${ }^{21,22}$. 
$20 \mu \mathrm{l}$ of the EOs was diluted with heptane to $10 \mathrm{ml}$ and the enantiomeric excess (ee) in \% was calculated from peak area (PA) by the following Eq. (1). The data were acquired with Chrom Card Trace Focus GC (Thermo Fisher scientific, version 2.9).

$$
e e(\%)=\frac{P A \text { of predominant enantiomer }-P A \text { of minor enantiomer }}{P A \text { of predominant enantiomer }+P A \text { of minor enantiomer }} \times 100
$$

Statistical analysis. The statistical analysis and illustration were carried out using GraphPad Prism 8 (version 8.0.0 (224)) software. Results were expressed as mean \pm standard deviation. Mean values were compared by either unpaired t-test (with Welch's correction when no homoscedasticity) or an ordinary one-way Welch's ANOVA test (with Welch's correction when no homoscedasticity) followed by Games-Howell's multiple comparisons post-hoc test. A $p$ value $<0.05$ was considered to be statistically significant. Prior to ANOVA, normal distribution using Shapiro-Wilk test $(\alpha=0.05)$ and homoscedasticity using Brown-Forsythe test $(p<0.05)$ were asserted. ChemDraw Professional (version 19.0.0.26) was used to generate the chemical structures. Principle component analysis (PCA) was performed on fourth root calculated data. The dataset was composed of the commercial $(n=11)$ and primary pine EOs $(n=54)$ characterized by 39 compounds and $( \pm)$ - $\alpha$-pinene (Supplementary Information, Table A3 and A4). PCA was performed with Rstudio (version 1.2.5019; packages: ggbiplot, version 0.55 ; pca3d, version 0.10 ).

Received: 31 March 2021; Accepted: 30 July 2021

Published online: 19 August 2021

\section{References}

1. European Pharmacopoeia (Ph. Eur.). European Directorate for the Quality of Medicines \& HealthCare EDQM (2020).

2. Konig, W. A. \& Hochmuth, D. H. Enantioselective gas chromatography in flavor and fragrance analysis: Strategies for the identification of known and unknown plant volatiles. J. Chromatogr. Sci. 42, 423-439. https://doi.org/10.1093/chromsci/42.8.423 (2004).

3. McConathy, J. \& Owens, M. J. Stereochemistry in drug action. Prim. Care Companion J. Clin. Psychiatry 5, 70-73. https://doi.org/ 10.4088/pcc.v05n0202 (2003).

4. Allenspach, M., Valder, C., Flamm, D., Grisoni, F. \& Steuer, C. Verification of chromatographic profile of primary essential oil of Pinus sylvestris L. combined with chemometric analysis. Molecules 25, 2973. https://doi.org/10.3390/molecules25132973 (2020).

5. Da Silva, A. C. et al. Biological activities of alpha-pinene and beta-pinene enantiomers. Molecules 17, 6305-6316. https://doi.org/ 10.3390/molecules17066305 (2012).

6. Rufino, A. T. et al. Anti-inflammatory and chondroprotective activity of (+)-a-pinene: structural and enantiomeric selectivity. J. Nat. Prod. 77, 264-269. https://doi.org/10.1021/np400828x (2014).

7. Michaelakis, A. et al. Citrus essential oils and four enantiomeric pinenes against Culex pipiens (Diptera: Culicidae). Parasitol. Res. 105, 769-773. https://doi.org/10.1007/s00436-009-1452-7 (2009).

8. Lebanov, L., Tedone, L., Kaykhaii, M., Linford, M. R. \& Paull, B. Multidimensional gas chromatography in essential oil analysis. Part 1: Technical developments. Chromatographia 82, 377-398. https://doi.org/10.1007/s10337-018-3649-3 (2019).

9. Konig, W. A., Fricke, C., Saritas, Y., Momeni, B. \& Hohenfeld, G. Adulteration or natural variability? Enantioselective gas chromatography in purity control of essential oils. HRC-J. High Resolut. Chromatogr. 20, 55-61. https://doi.org/10.1002/jhrc.1240200202 (1997).

10. Do, T. K. T., Hadji-Minaglou, F., Antoniotti, S. \& Fernandez, X. Authenticity of essential oils. TrAC Trends Anal. Chem. 66, 146-157. https://doi.org/10.1016/j.trac.2014.10.007 (2015).

11. Boren, K. E., Young, D. G., Woolley, C. L., Smith, B. L. \& Carlson, R. E. Detecting essential oil adulteration. J. Environ. Anal. Chem. https://doi.org/10.4172/jreac.1000132 (2015).

12. Baser, K. H. C. \& Buchbauer, G. Handbook of Essential Oils Science, Technology, and Applications 2nd edn. (CRC Press, 2016).

13. Beale, D. J., Morrison, P. D., Karpe, A. V. \& Dunn, M. S. Chemometric analysis of lavender essential oils using targeted and untargeted GC-MS acquired data for the rapid identification and characterization of oil quality. Molecules 22, 1339. https://doi.org/10. 3390/molecules22081339 (2017).

14. Schmidt, B. M. in Medicinal and Aromatic Crops: Production, Phytochemistry, and Utilization Vol. 1218 ACS Symposium Series Ch. 3, 27-48 (American Chemical Society, 2016).

15. ECHA. European Chemicals Agency: Pine, Pinus sylvestris, ext. https://echa.europa.eu/de/registration-dossier/-/registered-dossi er/17153/1/2.

16. Tisserand, R. \& Young, R. Essential oil safety 2nd edn. (Elsevier, 2014).

17. Hiltunen, R. \& Laakso, I. Gas chromatographic analysis and biogenetic relationships of monoterpene enantiomers in Scots pine and juniper needle oils. Flavour Fragr. J. 10, 203-210. https://doi.org/10.1002/ff.2730100314 (1995).

18. Ochocka, J. R., Asztemborska, M., Sybilska, D. \& Langa, W. Determination of enantiomers of terpenic hydrocarbons in essential oils obtained from species of Pinus and Abies. Pharm. Biol. 40, 395-399. https://doi.org/10.1076/phbi.40.5.395.8452 (2002).

19. Aguas, I., Alarcon, E. \& Villa, A. L. Turpentine valorization by its oxyfunctionalization to nopol through heterogeneous catalysis. Heliyon 6, e03887. https://doi.org/10.1016/j.heliyon.2020.e03887 (2020).

20. WHO guidelines on good agricultural and collection practices (GACP) for medicinal plants (2003).

21. Naspi, C., Costa, A. A., Lucia, A., Audino, P. G. \& Masuh, H. M. Enantiomeric ratio changes of terpenes in essential oils from hybrid Eucalyptus grandis x E. tereticornis and its pure species. J. Braz. Chem. Soc. 29, 419-429. https://doi.org/10.21577/0103-5053.20170 156 (2018).

22. Vandendool, H. \& Kratz, P. D. A generalization of the retention index system including linear temperature programmed gas-liquid partition chromatography. J. Chromatogr. 11, 463-471. https://doi.org/10.1016/s0021-9673(01)80947-x (1963).

\section{Acknowledgements}

The authors would like to thank Danielle Lüthi, Francesca Grisoni (Institute of Pharmaceutical Sciences, ETH Zurich) and the GMP-team of Systema Natura GmbH for valuable advice and sample provision, respectively. 


\section{Author contributions}

M.A. and D.F prepared and analyzed the EOs. C.V. and C.S. designed the study protocol. M.A. and C.S. interpreted all obtained data. M.A. and C.S. were major contributors in writing the manuscript. All authors reviewed the manuscript.

\section{Competing interests}

Systema Natura confirms in the name of all authors (C.V., D.F.) employed at Systema Natura GmbH, that this research may lead to the development of products in which Systema Natura has a business interest. This is in accordance with the policies of the company as well as in the interest of the individuals and their ethical obligation as a researcher. Accordingly, C.V. and D.F. have disclosed those interests fully to ETH Zürich. Systema Natura has established an approved plan for managing any potential conflicts arising from this arrangement. M.A. and C.S declare that there is no conflict of interests.

\section{Additional information}

Supplementary Information The online version contains supplementary material available at https://doi.org/ 10.1038/s41598-021-96356-x.

Correspondence and requests for materials should be addressed to C.S.

Reprints and permissions information is available at www.nature.com/reprints.

Publisher's note Springer Nature remains neutral with regard to jurisdictional claims in published maps and institutional affiliations.

Open Access This article is licensed under a Creative Commons Attribution 4.0 International License, which permits use, sharing, adaptation, distribution and reproduction in any medium or format, as long as you give appropriate credit to the original author(s) and the source, provide a link to the Creative Commons licence, and indicate if changes were made. The images or other third party material in this article are included in the article's Creative Commons licence, unless indicated otherwise in a credit line to the material. If material is not included in the article's Creative Commons licence and your intended use is not permitted by statutory regulation or exceeds the permitted use, you will need to obtain permission directly from the copyright holder. To view a copy of this licence, visit http://creativecommons.org/licenses/by/4.0/.

(C) The Author(s) 2021 